\title{
Visual alleys as a function of instructions under informative and reduced conditions of viewing
}

\author{
ATSUKI HIGASHIYAMA, TAKUMA ISHIKAWA, and KAORU TANAKA \\ University of Osaka Prefecture, Osaka, Japan
}

\begin{abstract}
Three experiments were designed to explore parallel and distance alleys as a function of instructions (apparent and objective) under three different distance-cue conditions. The main findings were that (1) the alleys for the apparent instructions were positioned closer to the median plane than those for the objective instructions, (2) in an indoor setting, the parallel and distance alleys were not different under either the apparent or the objective instructions, and (3) in an outdoor setting, the parallel alley lay inside the distance alley under both the apparent and the objective instructions. On the basis of comparison with some of the previous alley studies, it is suggested that exact control of instructions will not produce great discrepancy between the parallel and the distance alleys that were constructed indoors.
\end{abstract}

This paper concerns the visual alleys in which two series of points extending in depth at the subject's eye level are adjusted to appear as two straight lines parallel with one another (parallel alleys), or to appear equal in lateral separation (distance alleys). The physical loci of the visual alleys are generally far from actual parallel straight lines. Blumenfeld (1913), attempting to compare the two types of alleys, found the parallel alley to be positioned closer to the median plane than the distance alley was. Indow, Inoue, and Matsushima (1962) and Zajaczkowska (1956) confirmed Blumenfeld's results, and Indow and Watanabe (1984) have provided evidence that this relation between the parallel and distance alleys also holds true for moving points.

Hardy, Rand, and Rittler (1951) and Ishii (1972), on the other hand, found that about half of their subjects constructed the parallel alley inside the distance alley, but that the remaining subjects constructed the parallel alley outside the distance alley. Similar evidence was obtained in large open fields by Battro, di Pierro Netto, and Rozestraten (1976). These studies suggest that although there may be great individual differences, the mean setting of the parallel alleys should not differ from those of the distance alleys.

To explain these different outcomes of visual alleys, we explored the effects of instructions and distance cues on the loci of alleys. In Experiment 1, the effects of apparent and objective instructions were compared in total darkness, where primary distance cues such as accommodation, convergence, and disparity are available. The subjects adjusted the two types of alleys under each set of the instructions. Experiment 2 consisted of a replica

This study was suppported by a Grant-in-Aid for Scientific Research (No. 62710072) provided by the Japanese Ministry of Education, Science, and Culture. Correspondence may be addressed to Atsuki Higashiyama, Psychology Laboratory, University of Osaka Prefecture, Mozu-umemachi, Sakai, Osaka 591, Japan. of Experiment 1, done in a fully illuminated laboratory. Experiment 3 was an expansion of Experiment 2, done in a naturalistic open field.

\section{EXPERIMENT 1}

\section{Method}

Subjects. The subjects were 30 undergraduate students. No special qualifying criteria were imposed.

Apparatus. The apparatus consisted of five optical benches, which were arranged in parallel at different distances from the subject. On each bench there were two iron slides, each of which carried a light-emitting diode $1 \mathrm{~mm}$ in diameter. The farthest pair of lights (i.e., the standard pair of points) was fixed at a viewing distance of $437 \mathrm{~cm}$, with a lateral distance of $29.2 \mathrm{~cm}$ from the median plane to each point. The remaining four pairs of points were variable, at viewing distances of $302,194,107$, and $40 \mathrm{~cm}$. These pairs of points will be called the variables 1 through 4 , in that order.

Procedure. The subject's head was fixed with an adjustable headand chinrest, but eye movement was permitted freely. The light points were observed binocularly at the horizontal plane.

All subjects constructed the parallel and distance alleys. The adjustments of the variable points were made by the experimenter as directed by the subject. For the parallel alley, all points were simultaneously presented to the subject. The subject was first asked to direct attention to the standard and the variable 1 . The subject's task was to make lateral adjustments of the variable 1 , so that the line connecting the two left points would be parallel to the line between the two right points. After completing the variable 1, the subject adjusted the lateral separation of the variable 2, so that the left and right lines connecting from the standard through the variable 1 to the variable 2 were straight and parallel to one another. The variables 3 and 4 were likewise adjusted. After completing all variable points, the subject was asked to look over the entire alley and was allowed to require readjustments of any variable point.

For the distance alley, the standard pair and a variable pair were presented at the same time. The subject was first asked to adjust the lateral separation of the variable 1 so as to equal the lateral separation of the standard. After adjustments of the variable 1 , it was turned off and the variable 2 was turned on. The lateral separation of the variable 2 was then adjusted so as to equal that of the standard. The variables 3 and 4 were likewise adjusted. 
Half the subjects constructed the two types of alleys under the apparent instructions. The remaining subjects constructed them under the objective instructions. The adjustment order of the parallel and distance alleys was determined randomly for each subject. For each variable pair, an ascending trial and a descending trial were run in the sequence AD or DA. The lateral distance of each variable point from the median plane was read by the experimenter to an accuracy of $1 \mathrm{~mm}$.

Instructions. The apparent instructions for the parallel alley were:

We will show you some small lights. The position of the standard pair (i.e., the farthest pair of lights) will be fixed. Please arrange other closer pairs so that they appear to be on straight and parallel lines. We wish you to think not of where the lights actually are, but merely of how you sense them. By apparent parallel lines, we mean that two series of lights appear parallel to you, neither converging nor diverging. In this sense, a railroad track does not form apparent paralle! lines, because it appears to converge at the horizon. We are concerned with the two series of lights that do not appear to intersect even if they are imaginarily extended in front of or behind you.

The apparent instructions for the distance alley were:

We will show you two pairs of lights at a time. The position of the standard pair (i.e., the farthest pair of lights) will be fixed. Please arrange the lights of the variable pair so that the lateral distance between them appears to be the same as that between the standard pair. We wish you to judge on the basis of the apparent distance between the lights. By apparent distance, we mean an immediate, instantaneous impression of distance. Apparent distance may be equal to the actual distance, or it may not; we are not concerned with that.

The objective instructions for the parallel alley were:

We will show you some small lights. The position of the standard pair will be fixed. Please arrange other closer pairs so that they are on straight and parallel lines. We wish you to judge on the basis of the objective position of the lights. By objective parallel lines, we mean two straight lines, just like rails that never intersect in front of or behind you. Please construct the parallel alley as precisely as possible.

The objective instructions for the distance alley were:

We will show you two pairs of lights at a time. The position of the standard pair will be fixed. Please arrange the lights of the variable pair so that the lateral distance between them is the same as that between the standard pair. We wish you to judge on the basis of the objective distance between the lights. By objective distance, we mean the actual distance as measured with tape or stick. Please construct the distance alley as precisely as possible.

\section{Results}

Lateral distances from the median plane to the left and right points were obtained for each variable. This pair of values was converted into a single averaged value to use as a statistical score.

Figure 1 shows the main results of Experiment 1. Mean lateral distance is plotted as a function of viewing distance; the parameters comprise instruction and alley. Each data point represents a mean taken across trials and subjects $(N=30)$.

A four-way $(2 \times 2 \times 4 \times 2)$ mixed-model ANOVA was performed, in which instruction was a betweensubjects factor and alley, distance, and trial were withinsubjects factors.

The main effect of alley, the alley $\times$ distance interaction, and the alley $x$ distance $x$ instruction interaction were not significant. This means that the parallel and distance alleys were not significantly different at each viewing distance under each set of instructions.

The main effect of instruction was significant: Larger lateral distance was obtained for the objective instructions $(26.6 \mathrm{~cm})$ than for the apparent instructions $[15.8 \mathrm{~cm}$; $F(1,28)=33.7, p<.001]$.

The instruction $\times$ distance interaction was significant $[F(3,84)=35.1, p<.001]$. The simple main effect tests of this interaction showed that the lateral distance increased with increasing viewing distance for the apparent instructions $[F(3,84)=131.9, p<.001]$ and the objective instructions $[F(3,84)=11.9, p<.001]$. It was therefore suggested that this interaction was due to the

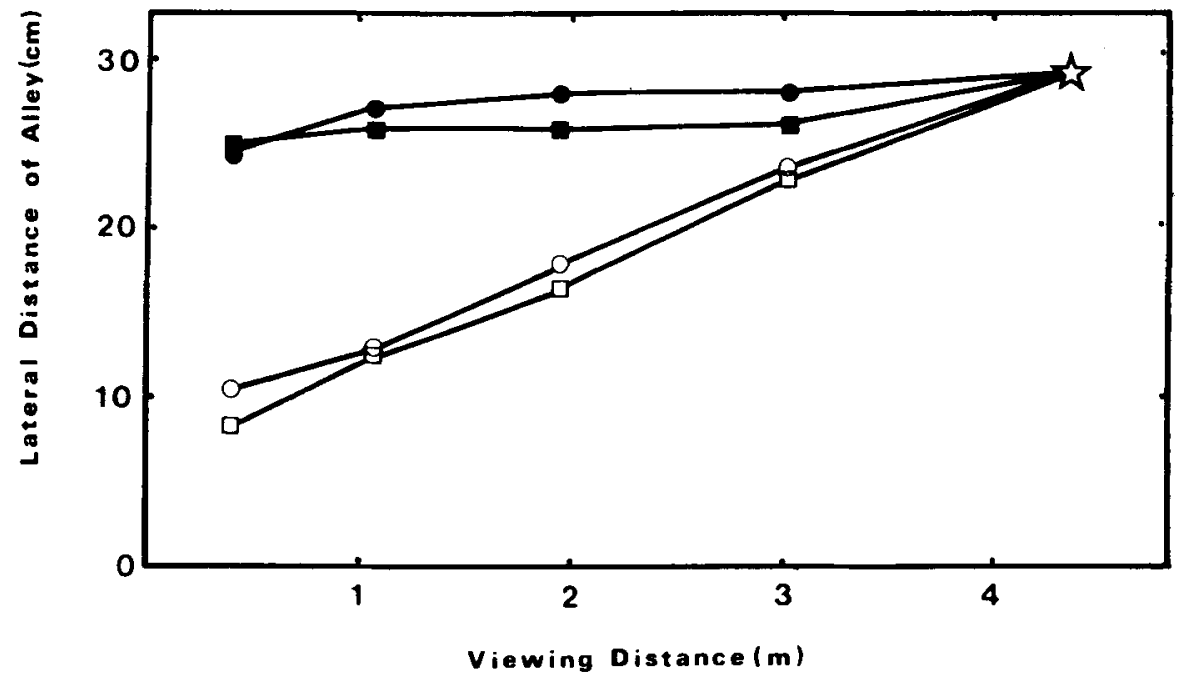

Figure 1. Mean lateral distance (in centimeters) from the median plane to visual alleys as a function of viewing distance (in meters). Open marks stand for the apparent instructions and filled marks for the objective instructions. Circles stand for the parallel alley and squares for the distance alley. A star stands for the standard point. 
steeper increase of lateral distance under the apparent instructions rather than under the objective instructions.

The main effect of trial was significant: The ascending trial $(18.8 \mathrm{~cm})$ produced smaller lateral distance than did the descending trial $[23.6 \mathrm{~cm} ; F(1,28)=19.3$, $p<.001]$.

The distance $\times$ trial interaction was significant $[F(3,84)=18.7, p<.001]$, as was the alley $\times$ distance $X$ trial interaction $[F(3,84)=3.64, p<.05]$. An analysis of simple interaction effects of this three-way interaction revealed that the simple distance $x$ trial interaction was significant for the parallel alley $[F(3,168)=$ $29.8, p<.001$ ], whereas it was not significant for the distance alley $(F<1)$. The differences between the two trials obtained for the viewing distances of $40,107,194$, and $302 \mathrm{~cm}$ were, respectively, $7.6,7.6,4.9$, and $1.8 \mathrm{~cm}$ for the parallel alley, and they were $4.8,5.3,3.7$, and $2.9 \mathrm{~cm}$ for the distance alley. Larger trial effects were thus suggested for closer variable points for the parallel alley.

\section{EXPERIMENT 2}

From the results of Experiment 1, it is clear that there was no significant difference between the loci of the parallel and distance alleys. The purpose in Experiment 2 was to examine the generality of the results of Experiment 1 by constructing the visual alleys in a fully illuminated laboratory.

\section{Method}

Experiment 2 was identical to Experiment 1, but for the following exceptions: (1) A dark-green wooden stick, $17.5 \mathrm{~cm}$ tall and $7 \mathrm{~mm}$ in diameter, was mounted on each slide on each optical bench, and the upper tips of the sticks were sharply pointed so as to focus the subject's attention on them; (2) the laboratory was illuminated by the fluorescent lamps on the ceiling, so that the subjects could see details of the equipment, such as the benches and tables, as well as the experimenter; (3) two groups of 14 subjects each, one of which received the apparent instructions and the other of which received the objective instructions, served as subjects. None of the subjects had participated in Experiment 1.

\section{Results}

The results are shown in Figure 2 in the same manner as in Figure 1. A four-way mixed-model ANOVA was performed as before.

The main effect of alley, the alley $x$ distance interaction, and the alley $x$ distance $x$ instruction interaction were not significant. This means that the parallel and distance alleys were not significantly different at each viewing distance under each set of instructions.

The main effect of instruction was significant: The objective instructions $(27.7 \mathrm{~cm})$ produced larger lateral distance than did the apparent instructions $[16.0 \mathrm{~cm}$; $F(1,26)=127.8, p<.001]$.

The instruction $x$ distance interaction was significant $[F(3,78)=61.1, p<.001]$. The simple main effect tests of this interaction showed that under the apparent instructions, the lateral distance increased with increasing viewing distance $[F(3,78)=94.1, p<.001]$, whereas under the objective instructions, it decreased slightly with increasing viewing distance $[F(3,78)=3.1, p<.05]$.

The main effect of trial was significant: The lateral distance for the ascending trial $(21.3 \mathrm{~cm})$ was smaller than that for the descending trial $[22.5 \mathrm{~cm} ; F(1,26)=20.0$, $p<.001]$.

The distance $\times$ trial interaction was also significant $[F(3,78)=9.6, p<.001]$. The differences between the

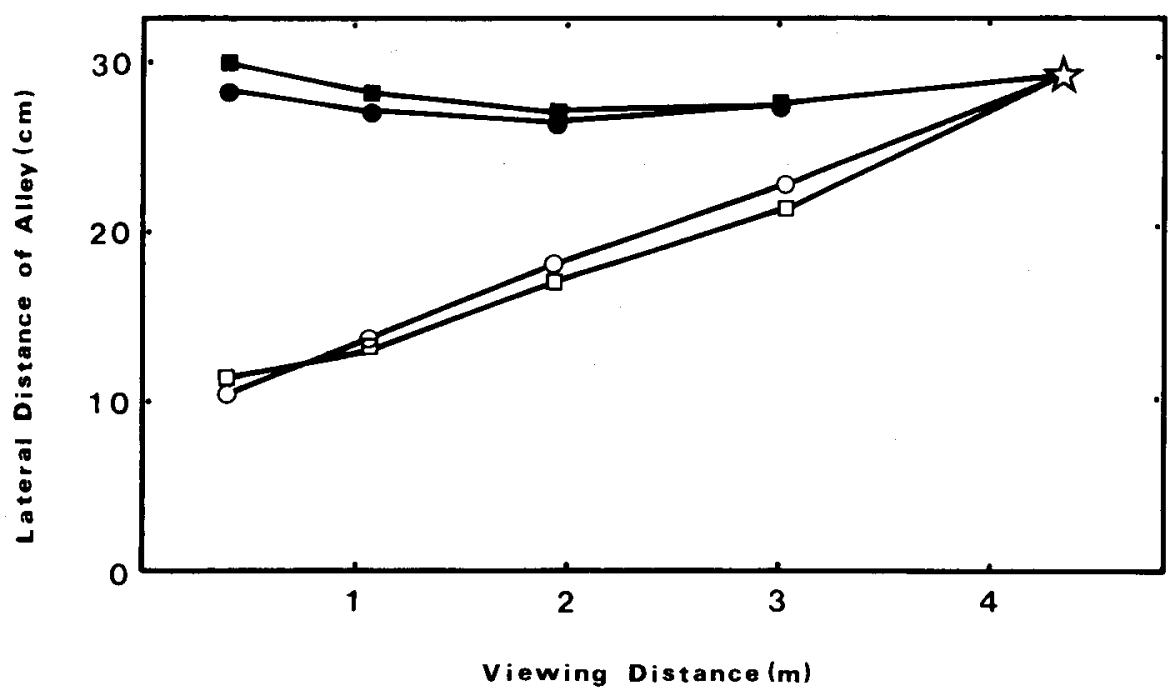

Figure 2. Mean lateral distance (in centimeters) from the median plane to visual alleys as a function of viewing distance (in meters). Open marks stand for the apparent instructions and filled marks for the objective instructions. Circles stand for the parallel alley and squares for the distance alley. A star stands for the standard point. 
two trials for the viewing distances of $40,107,194$, and $302 \mathrm{~cm}$ were, respectively, $1.9,1.3,1.1$, and $0.5 \mathrm{~cm}$. This suggests that significantly larger trial effects were found for closer variables.

\section{Intra- and Intersubject Variability of Judgments}

Since each subject performed an ascending and a descending trial for each variable, we were able to compute the difference between the two trials (an interval of uncertainty, IU), and the midpoint of this interval (a point of subjective equality, PSE).

The mean IU taken over the subjects may be a measure of intrasubject variability of judgments. The left portion of Figure 3 shows the mean IUs (descending minus ascending) obtained in Experiments 1 and 2 against viewing distance, with the parameters of instruction, alley, and distance cue. The standard deviation of the PSE data over the subjects may be a measure of intersubject variability of judgments. The right portion of Figure 3 shows the results in the same way as for the IU data. Both measures of variability seem very similar to one another: (1) The variability of judgments generally increases as viewing distance decreases; (2) it is larger for the reduced distance-cue condition than for the rich distance-cue condition; and (3) for closer distances under the reduced distance-cue condition, the parallel alley is likely to produce larger variability than that produced by the distance alley.

\section{EXPERIMENT 3}

From the results of Experiment 2, it is evident that the loci of the parallel and distance alleys were not signifi- cantly different in the fully illuminated laboratory. Taken together with the results of Experiment 1, this suggests that under the particular instructions, the parallel alley would be generally congruent with the distance alley, and the richness of distance information would not greatly influence the relative position of the alleys. However, these results may be due to the short viewing distances in the indoor settings. It is therefore possible that the effects of the subtle differences between the parallel- and distancealley instructions might manifest themselves in large viewing distances.

\section{Method}

In Experiment 3, two groups of 13 subjects each, one of which received the apparent instructions and the other of which received the objective instructions, attempted to construct the visual alleys on the campus lawn. Silver duralumin poles, $1 \mathrm{~m}$ tall and $2.5 \mathrm{~cm}$ in diameter, were placed on the ground as stimulus targets. The viewing distance of the standard pair of poles was $45 \mathrm{~m}$ and the lateral distance was $6 \mathrm{~m}$, so that the visual angle subtended by the poles was the same as that used in the indoor experiments. The viewing distances of four variable pairs of poles were $5,10,18$, and $29 \mathrm{~m}$. The experimenter varied the lateral separations of the variable pairs in accordance with the directions given by the subject. The lateral distance of each variable pole from the median plane was measured to an accuracy of $10 \mathrm{~cm}$. None of the subjects had participated in Experiment 1 or Experiment 2.

\section{Results}

The results are shown in Figure 4, where mean lateral distance of each variable pole from the median plane $(N=$ $26)$ is plotted against viewing distance, with instruction and alley as parameters.

A four-way $(2 \times 2 \times 4 \times 2)$ mixed-model ANOVA was performed as before. The main effect of instruction
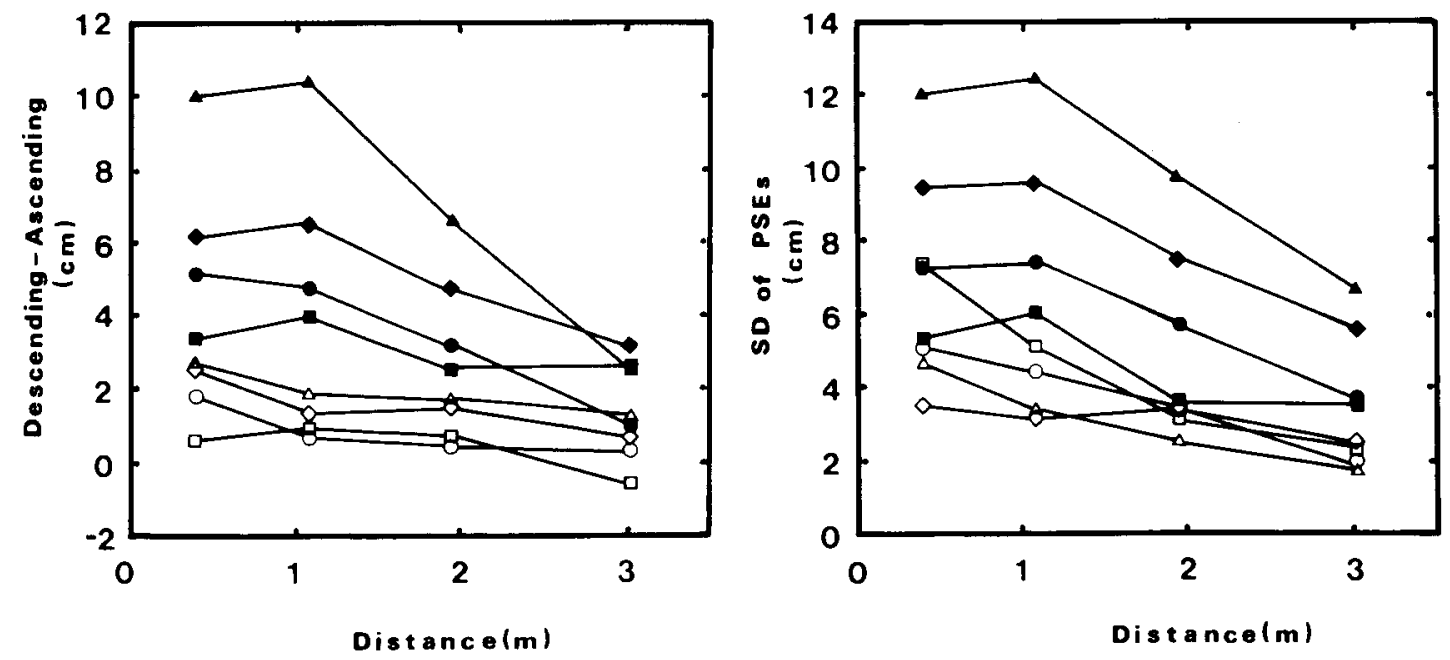

Figure 3. Variability of judgments (in centimeters) in Experiments 1 and 2 as a function of viewing distance (in meters). The left panel shows intrasubject variability (i.e., mean differences between descending and ascending trials), whereas the right portion shows intersubject variability (i.e., standard deviations of the PSEs over the subjects). Filled marks stand for the dark conditions and open marks for illuminated conditions. Circles stand for the apparent parallel, squares for the apparent distance, triangles for the objective parallel, and diamonds for the objective distance. 


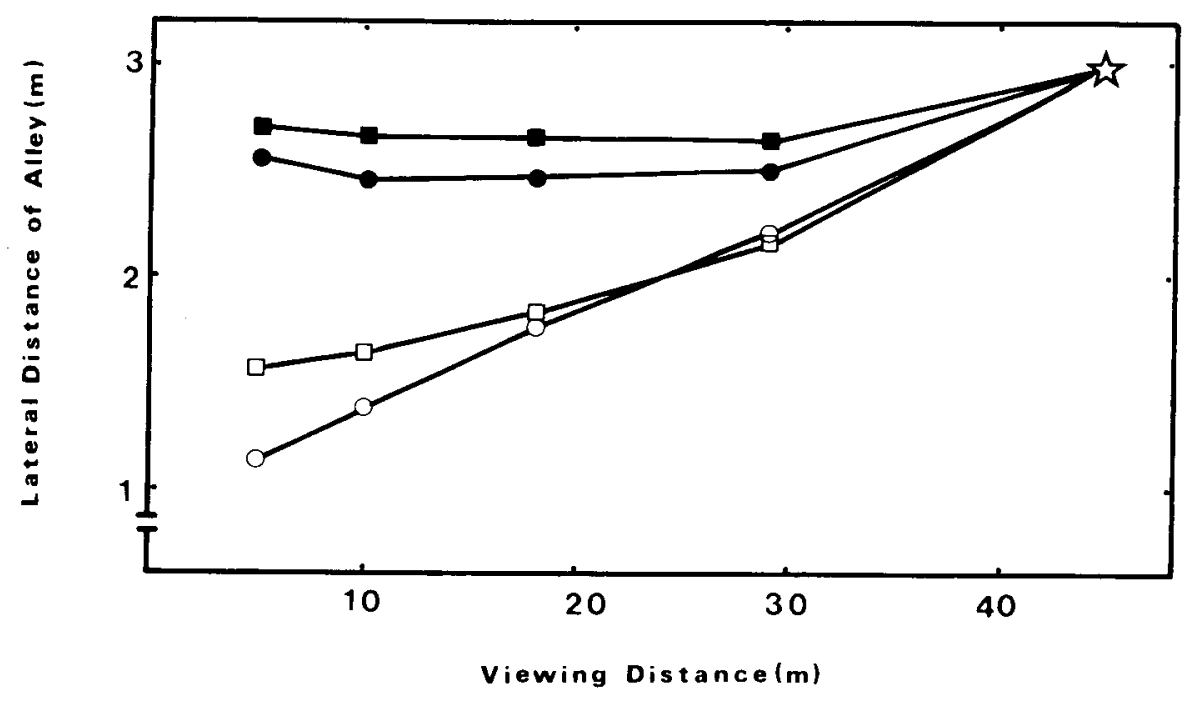

Figure 4. Mean lateral distance (in meters) from the median plane to visual alleys as a function of viewing distance (in meters). Open marks stand for the apparent instructions and filled marks for the objective instructions. Circles stand for the parallel alley and squares for the distance alley. A star stands for the standard pole.

was significant: The objective instructions $(2.6 \mathrm{~m})$ produced larger lateral distance than did the apparent instructions $[1.7 \mathrm{~m} ; F(1,24)=25.2, p<.001]$.

The instruction $\times$ distance interaction was also significant $[F(3,37)=22.2, p<.001]$. The simple main effect tests of this interaction showed that under the apparent instructions, the lateral distance increased with increasing viewing distance $[F(3,72)=43.5, p<.001]$, whereas under the objective instructions, it remained constant over the viewing distance $(F<1)$.

The main effect of alley was significant: The parallel alley lay inside the distance alley $[F(1,24)=7.7$, $p<.01]$.

The alley $x$ distance interaction was significant $[F(3,72)=4.6, p<.001]$, as was the instruction $\times$ alley $\times$ distance interaction $[F(3,72)=4.9, p<.001]$. An analysis of simple interaction effects of this three-way interaction indicated that the simple alley $x$ distance interaction was significant under the apparent instructions $[F(3,72)=9.3, p<.001]$, but that it was not significant under the objective instructions $(F<1)$. This means that under the apparent instructions, the difference between the parallel and distance alleys was greater for closer viewing distances, but under the objective instructions, it was constant regardless of viewing distance.

The tests of simple main effects of the instruction $x$ alley $x$ distance interaction indicated that under the apparent instructions, the parallel and distance alleys were significantly different only for viewing distances of $5 \mathrm{~m}$ $[F(1,72)=42.2, p<.001]$ and $10 \mathrm{~m}[F(1,72)=14.2$, $p<.001]$, and that under the objective instructions, they were significantly different for all viewing distances $(F \mathrm{~S}>3.9, p<.05)$.
The main effect of trial was significant: The ascending trial $(2.1 \mathrm{~m})$ was significantly smaller than the descending trial $[2.2 \mathrm{~m} ; F(1,24)=17.7, p<.001]$.

The distance $\times$ trial interaction was significant $[F(3,72)=6.9, p<.001]$, as was the alley $\times$ distance $\times$ trial interaction $[F(3,72)=5.3, p<.001]$. The simple distance $\times$ trial interaction was significant for the parallel alley $[F(3,144)=12.9, p<.001]$, but it was not significant for the distance alley $(F<1)$. The differences between the two trials obtained for the viewing distances of $5,10,18$, and $29 \mathrm{~m}$ were, respectively, 21,14 , 5 , and $4 \mathrm{~cm}$ for the parallel alley, and they were 11,11 , 10 , and $17 \mathrm{~cm}$ for the distance alley.

\section{GENERAL DISCUSSION}

\section{Loci of Alleys}

We obtained three findings regarding the loci of visual alleys. First, the alleys for the objective instructions approximated a physically parallel alley, and the alleys for the apparent instructions were positioned closer to the median plane than were those for the objective instructions. To be more specific, for the apparent instructions, the lateral distance of the alleys increased monotonically as the viewing distance increased, whereas, for the objective instructions, it increased (Experiment 1), decreased (Experiment 2), or remained constant with viewing distance (Experiment 3).

Second, in the indoor setting, under both the apparent and objective instructions, the loci of the parallel alley were not significantly different from those of the distance alley. This agrees with Hardy et al. (1951) and Ishii (1972), but not with Blumenfeld (1913), Indow et al. 
(1962), Indow and Watanabe (1984), and Zajaczkowska (1956). On the other hand, in the outdoor setting, the parallel alley lay inside the distance alley under both the apparent and the objective instructions. It thus appears that the discrepancy between the parallel and distance alleys is by no means the rule, and that it occurs only in a large open field.

One may here ask what caused the parallel and distance alleys to be congruent indoors but discrepant outdoors. A tentative interpretation is that the subtle differences between the parallel and distance alleys are amplified in the large open field. A major feature distinguishing the outdoor condition from the indoor condition was the total length of the alleys. For the indoor condition, the length was about $4.4 \mathrm{~m}$, whereas for the outdoor condition, it was about $45 \mathrm{~m}$. Therefore, if the difference between the parallel and distance alleys is magnified as a function of total length of the alleys, the discrepancy of the alleys is probably larger for the outdoor setting than for the indoor setting.

From the results for the indoor settings of the alleys, one may argue that most subjects could not appreciate the differences between the parallel- and distance-alley instructions, and, as a result, that they may have constructed the same alley under the two sets of instructions. However, there seem to be two reasons to believe that the subjects understood the instructions correctly. First, for closer distances under the reduced distance-cue condition, the intra- and intersubject variabilities were consistently larger for the parallel alley than for the distance alley. This suggests that the parallel alley is more difficult to construct than the distance alley. Second, in Experiment 3 , where the loci of the two alleys were significantly different, it is clear that such results would not be obtained without appreciation of the instructions.

Our final finding is that richness of distance cues does not greatly affect the loci of the alleys. This can be illustrated well through comparison of the results of Experiments 1 and 2 (Figures 1 and 2), which varied only in the distance cues available to the subjects. Somewhat similar results were obtained by Indow and Watanabe (1984), who showed that the loci of the alleys are not affected by whether the laboratory is dark or illuminated, or by whether stimulus points are stationary or moving. However, richness of distance cues contributed to reduction of intra- and intersubject variability of judgments. As is shown in Figure 3, the variabilities of the illuminated conditions are about a fourth to a half as small as those of the dark conditions.

It appears that, in making parallel and equidistance judgments, the dark-indoor subjects relied on a natural combination of primary distance cues and the illuminatedindoor subjects utilized both primary and pictorial distance cues (i.e., overlapping, texture gradient, light and shade, and so on). This difference in distance cues, taken with our final finding, suggests that the two sets of distance cues have the same effects on mean settings of the alleys, but that the alleys based on primary and pictorial distance cues are more stable than the alleys based on primary distance cues alone. In this case, therefore, pictorial distance cues may contribute to the stability rather than the locus of the visual alleys.

This may throw general doubt on the conventional view that distance cues always convey information about perceived distance. Our data suggest that some pictorial distance cues are probably more useful in the attainment of stability of perceived distance, rather than perceived distance in itself.

\section{Comparisons with Previous Alley Studies}

No study of the alleys has ever been designed to make a direct comparison between the apparent and the objective instructions. This is in remarkable contrast with studies of size perception, in which instructions are an important factor affecting size judgments (see Carlson, 1977 , for a recent review). However, the paradigm of the distance-alley experiment is essentially the same as the size-match procedure in which the subjects compare a far standard and a close variable (e.g., see Ehrenstein, 1977; Higashiyama, 1976; Ishii, 1972). Accordingly, it is probable that the locus of the distance alley varies greatly with instructions to the subjects. The various outcomes obtained in previous alley studies may be due to a failure to regulate the distance-alley instructions given to the subjects.

In some studies (Blumenfeld, 1913; Squires, 1956; Zajaczkowska, 1955), individual data have been provided for the specific instructions to the subjects. Blumenfeld (1913) had 7 subjects construct visual alleys under the apparent instructions. ' Figure 5 shows the results of the left alleys that were observed binocularly in total darkness. The parameter consists of the subjects. Each data point was a mean taken across various conditions, such as simultaneous versus successive presentation of points, point level at or above the eyes, and so forth.

It seems clear that the data points for the distance alley are scattered more widely than those for the parallel alley. This contrasts with our results, which showed that intra- and intersubject variability of judgments was smaller for the distance alley than for the parallel alley.

This may mean that the apparent instructions may have been understood differently by different subjects. In fact, there seem to be two typical distance alleys in Figure 5. The width of the alleys for some subjects (as marked by filled triangles, filled diamonds, and open circles) increases monotonically with the viewing distance, which is similar to what was found with the apparent distance alley in this study. On the other hand, the width of the alleys for other subjects (as marked by open squares and open diamonds) remains nearly constant up to about $2 \mathrm{~m}$ and increases with greater distance. This response pattern may suggest a mixture of the apparent and objective 

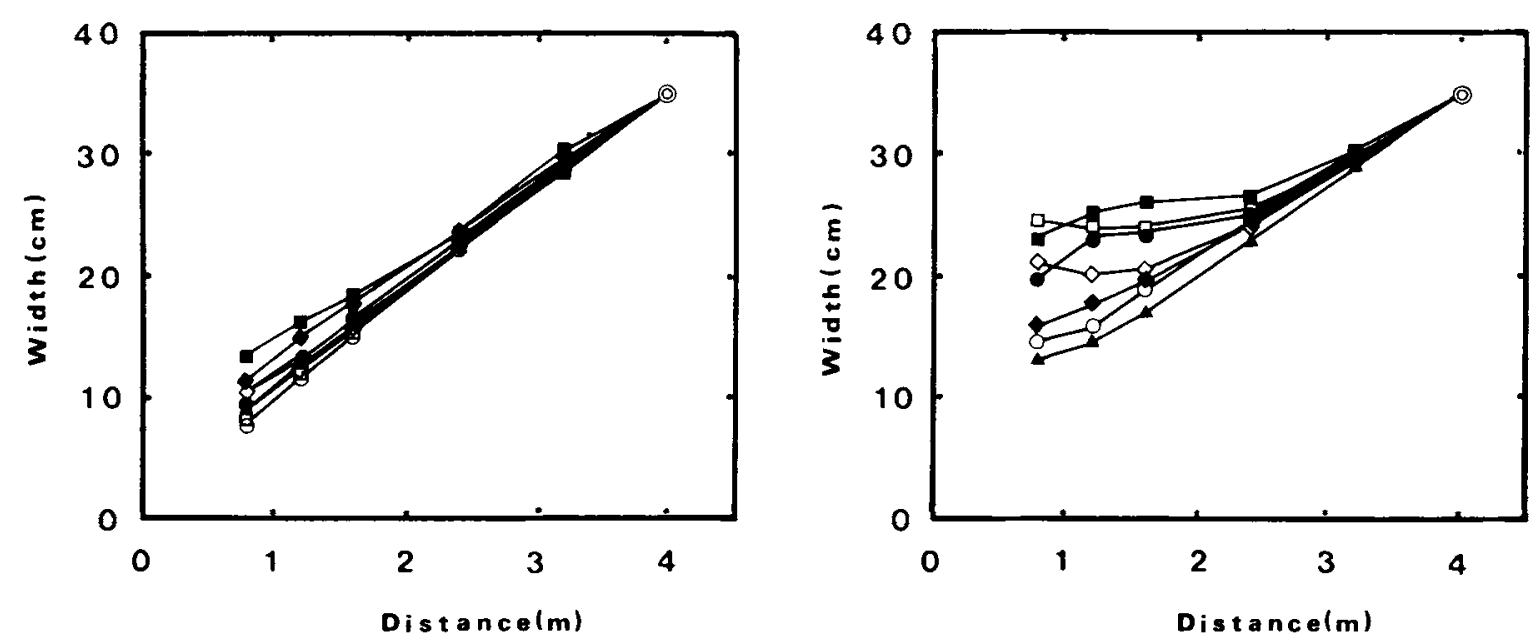

Figure 5. The parallel (left) and distance (right) alleys from Blumenfeld's (1913) data. The lateral distance (in centimeters) from the median plane to the left alleys is plotted against viewing distance (in meters). The parameter is the subjects. A double circle stands for the standard point.

distance alleys in Blumenfeld's (1913) study - that is, the objective attitude for nearby distances and the apparent attitude for far distances.

Zajaczkowska (1956) also described the instructions for the parallel and distance alleys. ${ }^{2}$ However, her instructions may have been ambiguous, because it is not clear whether her "two parallel lines" or "two distances"' are apparent or objective in nature. Zajaczkowska may not have distinguished between the apparent and objective instructions.

Figure 6 shows the results of Zajaczkowska's (1955, Appendix 5) "classical" alley experiments, in which 9 subjects constructed alleys binocularly in total darkness. The parameter consists of the subjects. Each data point is the mean lateral distance from the median plane to the left and right alleys. It also seems clear that the individual differences for the distance alley are greater than those for the parallel alley, particularly at closer distances. It appears that the instructions for the distance alley may not have been uniformly understood by the subjects.

It appears that the individual distance alleys illustrated in Figures 5 and 6 suggest in general the combinational responses of the alleys constructed with pure apparent and objective attitudes. The width of the individual distance alleys, $D$, may therefore be described by an averaging model (e.g., Anderson, 1974; Foley, 1977):

$$
D=W_{\mathrm{A}} D_{\mathrm{A}}+W_{\mathrm{o}} D_{\mathrm{o}}
$$

where $D_{\mathrm{A}}$ and $D_{\mathrm{o}}$ are, respectively, the widths of the alleys with the apparent and objective attitudes, and $W_{\mathrm{A}}$ and $W_{\mathrm{O}}$ are the weights assigned to each distance alley.

Squires (1956) had 4 subjects construct visual alleys in an illuminated room. His instructions for the parallel alley resembled Blumenfeld's, but the instructions for the distance alley seem to have required the subjects to compare any two apparent lateral distances using a "depth- perception attitude." Three of the subjects, in accordance with the instructions, succeeded to compare the lateral distances "as a unified depth-Gestalt (unitary attitude)," but 1 subject compared them "as separate configurations, not tied together in a depth-Gestalt (nonunitary attitude)" (pp. 291-293).

Figure 7 illustrates Squires' (1956) results, in which mean settings of the parallel alley $(N=4)$ are shown together with the settings of the unitary $(N=3)$ and nonunitary $(N=1)$ distance alleys. From Figure 7 , it is clear that the nonunitary distance alley hovered around the standard lateral distance of $10 \mathrm{~cm}$. This is very similar to the objective distance alley in this study. We think that the nonunitary attitude is substantially equivalent to the objective attitude, because, as Squires mentioned, the nonunitary attitude is "a purely analytic one" in building the distance alley, and, therefore, the nonunitary distance alleys are "not phenomenal realities but psychological abstractions" (p. 295).

It is also clear from Figure 7 that the width of the unitary distance alley generally increased with increasing viewing distance. This finding agrees with that for our apparent distance alley. It is, therefore, very likely that the unitary attitude has the same effects as the apparent attitude in the construction of the distance alley.

However, one may still wonder why, in the Squires (1956) study, the parallel alley lay outside the unitary distance alley, which may contrast with Blumenfeld's (1913), Zajaczkowska's $(1955,1956)$, and our results. This difference may be due mainly to visual angle subsumed by the standard points: Squires' visual angle of the standard points was about one third as small as the visual angles employed in the other studies mentioned above. Zajaczkowska (1956) showed that although the parallel alley lay inside the distance alley, this discrepancy between the two alleys was smaller for a smaller visual angle of the stan- 

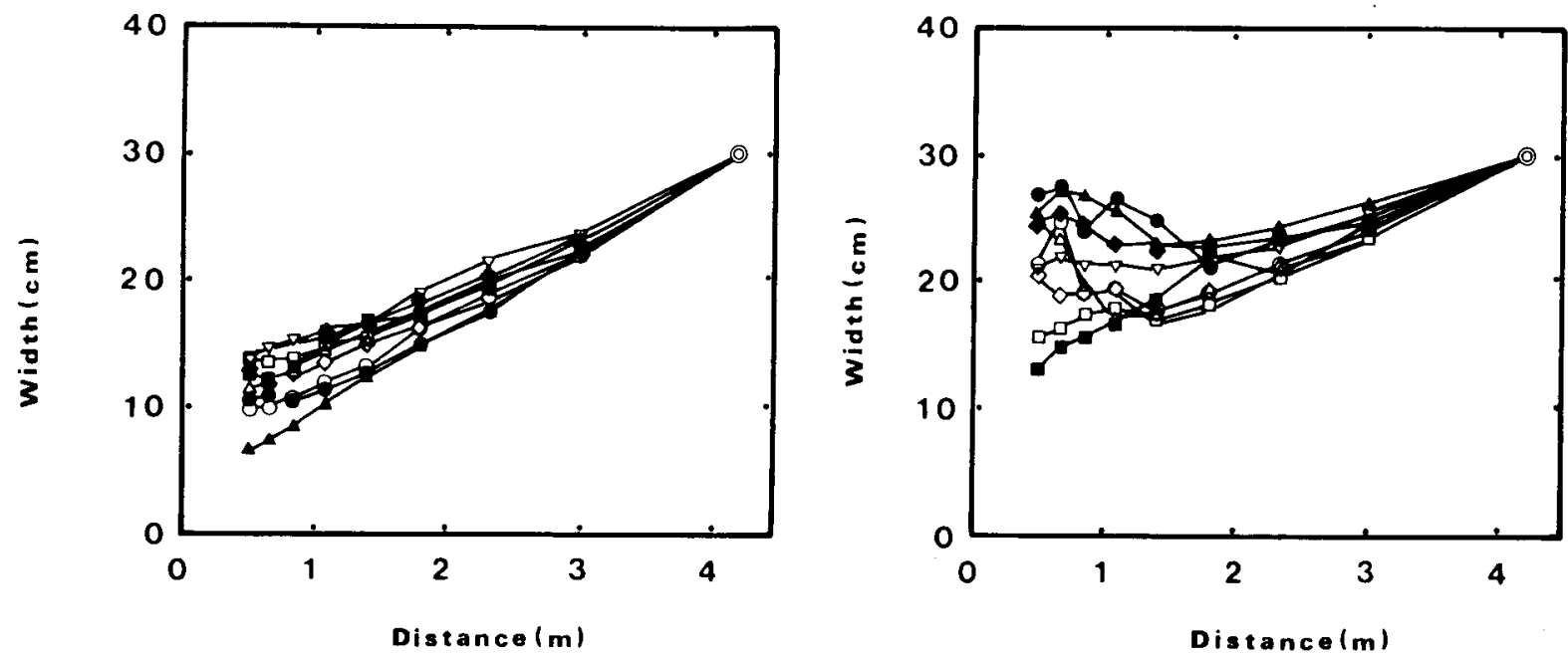

Figure 6. The parallel (left) and distance (right) alleys from Zajaczkowska's (1955) data. The mean lateral distance (in centimeters) from the median plane to left and right alleys is plotted against viewing distance (in meters). The parameter is the subjects. A double circle stands for the standard point.

dard points. Furthermore, Foley (1968) and Higashiyama $(1976,1981,1984)$ found that for a small configuration, the curvature of the space tends to be positive. This means that the parallel alley would lie outside the distance alley for a small visual angle of the standard points.

\section{Implications for the Geometry of Visual Space}

Luneburg $(1947,1950)$, assuming that binocular visual space is a Riemannian space of constant curvature (i.e., a homogeneous space), predicted that the loci of visual alleys vary as a function of curvature: For negative constant curvature (i.e., hyperbolic geometry), the parallel alley lies inside the distance alley; for zero curvature (i.e., Euclidean), both alleys are congruent with one another; for positive constant curvature (i.e., elliptic geometry), the parallel alley lies outside the distance alley. Thus, it

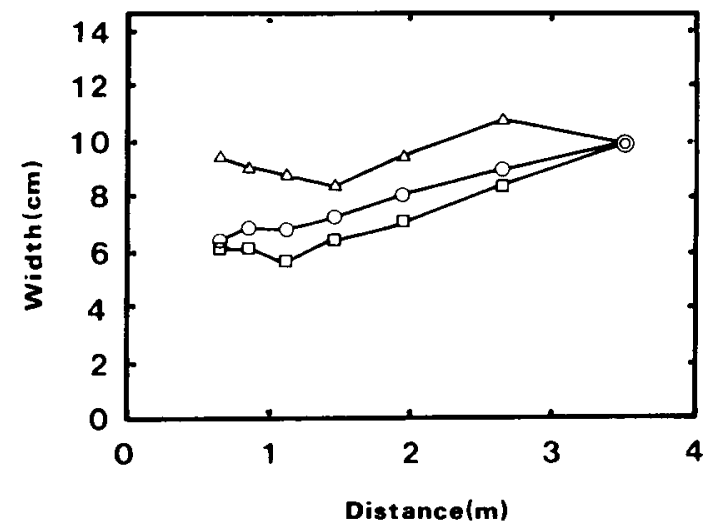

Figure 7. The parallel and distance alleys from Squires' (1956) data. The circles stand for the parallel alley; squares, for the distance alley with unitary attitude; triangles, for the distance alley with nonunitary attitude. may be concluded that the indoor space is Euclidean, but the outdoor space is hyperbolic.

However, recent studies of binocular visual space have thrown strong doubt on the assumptions that visual space is homogeneous (Foley, 1972). In particular, Higashiyama $(1981,1984)$ has shown that although curvature may remain constant within a given configuration, it varies with the visual angle of the stimulus configuration: The larger the visual angle, the more the curvature tends to be negative. This finding has an important implication for the alley configuration, the visual angle of which becomes larger as the variable approaches the subject. That is, the curvature is more negative for the variable closer to the subject. Moreover, Wagner (1985) reported failure of Luneburg's model in describing the data of distance, angle, and area in a large open field. It is therefore apparent that the geometry of visual space is not as simple as Luneburg postulated. In this sense, we cannot determine the geometry of visual space on the basis of alley experiments alone.

\section{REFERENCES}

ANDERson, N. H. (1974). Algebraic models in perception. In E. C. Carterette \& M. P. Freedman (Eds.), Handbook of perception (Vol. 2, pp. 215-298). New York: Academic Press.

Battro, A. M., di Pierro Netto, S., Rozestraten, R. J. (1976). Riemannian geometries of variable curvature in visual space: Visual alleys, horopters, and triangles in big open fields. Perception, 5, 9-23.

BLumENFELD, W. (1913). Untersuchungen über die scheinbare Grösse im Sehraume. Zeitschrift fur Psychologie, 65, 241-404.

CARLSON, V. R. (1977). Instructions and perceptual constancy judgments. In W. Epstein (Ed.), Stability and constancy in visual perception: Mechanisms and processes (pp. 217-254). New York: Wiley.

EHRENSTEIN, W. H. (1977). Geometry in visual space-some methoddependent (arti)facts. Perception, 6, 657-660.

Foley, J. M. (1968). Depth, size and distance in stereoscopic vision. Perception \& Psychophysics, 3, 265-274.

Foley, J. M. (1972). The size-distance relation and intrinsic geometry 
of visual space: Implications for processing. Vision Research, 12, 323-332.

FoLEY, J. M. (1977). Effect of distance information and range on two indices of visually perceived distance. Perception, 6, 449-460.

Hardy, L. H., RAND, G., \& Ritiler, A. C. (1951). Investigation of visual space: The Blumenfeld alley. A.M.A. Archives of Ophthalmology, 45, 53-63.

Higashiyama, A. (1976). The 3- and 4-point experiments and a test of homogeneity in binocular visual space. Japanese Journal of Psychology, 47, 149-157.

Higashiyama, A. (1981). Variation of curvature in binocular visual space estimated by the triangle method. Vision Research, 21, 925-933.

Higashiy ama, A. (1984). Curvature of binocular visual space: A modified method of right triangle. Vision Research, 24, 1713-1718.

InDow, T., Inoue, E., Matsushima, K. (1962). An experimental study of the Luneburg theory of binocular space (2): The alley experiments. Japanese Psychological Research, 4, 17-24.

Indow, T., \& Watanabe, T. (1984). Parallel-and distance-alleys with moving points in the horizontal plane. Perception \& Psychophysics, 35, 144-154.

IsHII, K. (1972). Sho-koujougenshou kan no soukan [Studies on the correlations among various kinds of constancy]. In Y. Akishige (Ed.), Chikaku-teki sekai no koujousei: Ninshiki shinrigaku [Constancies of perceptual world: Epistemological psychology] (Vol. 4, pp. 21-198). Tokyo: Ibunsha.

LUNEBURG, R. K. (1947). Mathematical analysis of binocular vision. Princeton, NJ: Princeton University Press.

Luneburg, R. K. (1950). The metric of binocular visual space. Journal of the Optical Society of America, 40, 627-642.

SQuires, P. C. (1956). Luneburg theory of visual geodesics in binocular space perception. A.M.A. Archives of Ophthalmology, 56, 288-297.

Wagner, M. (1985). The metric of visual space. Perception \& Psychophysics, 38, 483-495.

ZAJACZKOWSKA, A. (1955). An experimental study of Luneburg's theory of visual perception. Unpublished doctoral dissertation, University of London.
ZAJACZKowSKA, A. (1956). Experimental test of Luneburg's theory: Horopter and alley experiments. Journal of the Optical Society of America, 46, 514-527.

\section{NOTES}

1. Blumenfeld's (1913) instructions for the parallel alley were:

Please adjust the flames (sticks), so that the lines connecting them with the farthest flames (sticks) appear to you as parallel and symmetric lines-that is, the two lines neither converge nor diverge in front and in the rear. The observation should be made with eye movement along the lines under the usual viewing attitude (p. 286-287).

The instructions for the distance alley were:

Please adjust the light, so that the lateral distance of each pair of flames appears to you to be equal to that of the farthest pair. It is not necessary that the distance of a variable pair appear equal to that of another variable pair. The lines extending in depth by connecting lights should not be taken into account; the form of this curve is not important at all. Please do your best so as to make symmetric adjustments. In making distance comparisons, you should not use absolute values of a measuring stick with objective units such as $\mathrm{cm}$ or $\mathrm{m}$ (p. 310).

2. The instructions for the parallel alley were: "Form two parallel lines. They should neither converge nor diverge. Pay attention to the lines as a whole. The lines should be straight, of course, but first of all they must be parallel"'; the instructions for the distance alley were: "Look at the two end lights. How far is it from the left light to the right light? Then look at the new lights. How far is it from the left light to the right light? Make these two distances equal."

(Manuscript received July 13, 1989; revision accepted for publication November 13, 1989.) 